Théologiques

Théologiques

\title{
La foi ( pistis ) entre croyance et savoir selon Origène dans le Contre Celse
}

\section{Alain Le Boulluec}

Volume 13, numéro 1, printemps 2005

Croire et croyances

URI : https://id.erudit.org/iderudit/012525ar

DOI : https://doi.org/10.7202/012525ar

Aller au sommaire du numéro

\section{Éditeur(s)}

Faculté de théologie et de sciences des religions, Université de Montréal

ISSN

1188-7109 (imprimé)

1492-1413 (numérique)

Découvrir la revue

Citer cet article

Le Boulluec, A. (2005). La foi ( pistis ) entre croyance et savoir selon Origène dans le Contre Celse. Théologiques, 13(1), 59-78.

https://doi.org/10.7202/012525ar
Résumé de l'article

Laissant de côté le pacte entre contractants de puissance inégale que supposent les termes pistis et pisteuein et l'élément quasi institutionnel qui établit la foi ( pistis ) sur une règle ecclésiastique, l'enquête s'applique à la réponse d'Origène au pamphlet du philosophe Celse contre le christianisme. À Celse qui réduit la foi chrétienne à la croyance et qui la soumet donc au doute, Origène prétend opposer les garants qui assurent sa validité, selon les méthodes de la persuasion rhétorique. Il l'éloigne de la croyance et de la coutume en lui octroyant un fondement " naturel ». Il est conduit aussi à admettre plusieurs degrés dans la connaissance religieuse, en modifiant la distinction opérée par Platon entre « foi » et « intellec-tion ». L'exigence de compréhension et de savoir cependant n'exclut pas, dans la recherche du sens des Écritures comme dans les rapports avec les puissances invisibles, le recours à des pratiques de type rituel qui impliquent une expérience étrangère au régime de la croyance et de la foi.
Tous droits réservés ( Faculté de théologie et de sciences des religions, Université de Montréal, 2006
Ce document est protégé par la loi sur le droit d'auteur. L'utilisation des services d’Érudit (y compris la reproduction) est assujettie à sa politique d'utilisation que vous pouvez consulter en ligne.

https://apropos.erudit.org/fr/usagers/politique-dutilisation/ 


\title{
La foi (pistis) entre croyance et savoir selon Origène dans le Contre Celse
}

\author{
Alain Le BoullueC \\ École Pratique des Hautes Études (ÉPHÉ) \\ Sorbonne, Paris
}

\section{Introduction}

Alors qu'en latin une relation supplétive s'est instaurée entre le verbe credo et le substantif fides, la racine *bheidh en grec a fourni deux couples associés, d'une part le verbe peithomai ( «être persuadé, avoir confiance, obéir») et le substantif abstrait peithô ( "persuasion») et d'autre part le verbe pisteuô («avoir foi ») et le nom d'action pistis ( «confiance, foi »). Les implications juridiques de la signification originelle de cette famille, qui ont été repérées par Émile Benveniste $(1969,115-121)$ à propos du latin fides, sont perceptibles en grec. Le christianisme a fait évoluer pistis vers le sens de «foi religieuse» et pisteuô vers celui de "confesser sa pistis », à tel point que pistis peut désigner la somme de ce qui doit être cru et même le symbole de foi, l'ensemble des articles du credo (voir Lampe 1961, «Pistis », III, 1087). Ces termes supposent un pacte entre contractants de puissance inégale qui, dans le christianisme, a donné une expression particulièrement forte à l'autorité qui s'exerce et à la protection sur celui qui s'y soumet, en échange et dans la mesure de sa soumission ${ }^{1}$, en lien avec la doctrine du salut.

Mon propos est ici de mettre en évidence quelques aspects de la pistis chrétienne, telle qu'elle est définie par Origène dans sa réplique au pamphlet du philosophe Celse contre le christianisme². Je laisserai de côté l'élément quasi institutionnel qui établit la pistis sur une règle ecclésiastique, tel

1. Selon les termes de Benveniste (1969) à propos des relations établies entre les partenaires de la confiance par la fides latine.

2. Les travaux sur la foi selon Origène sont nombreux, et ils sont répertoriés, parfois analysés dans la Bibliographie critique d'Origène de Henri Crouzel (1971; 1982; 1996). 
qu'il apparaît par exemple dans la préface du Traité des principes. Je n'examinerai pas tous les arguments de la réfutation d'Origène qui veulent associer la foi à la raison en dénonçant l'inanité des griefs de l'adversaire. Ils ont été répertoriés et analysés par Lorenzo Perrone (1998) dans le commentaire qu'il a donné de Contre Celse, I,9-13. Je ne reviendrai pas non plus sur l'usage très précis que fait Origène de la logique et de l'épistémologie stoïciennes pour invalider les objections de Celse et pour donner valeur d'évidence aux propositions qu'il lui oppose. Les démonstrations de J.M. Rist (1981) et de H.J. Horn (1993) ont fait toute la lumière sur ce point.

J'examinerai d'abord (1) comment Origène assoit la crédibilité objective de la foi chrétienne en présentant les garants de celle-ci et ensuite (2) comment il éloigne la pistis de la croyance en la mettant au-dessus de ce qui est de l'ordre du nomizein, du nomos et de la doxa. J'essaierai aussi (3) de discerner sa propre perception de la pistis, le questionnement subjectif, d'inspiration à la fois philosophique et religieuse, auquel il la soumet.

La construction élaborée par Origène a contribué à imposer un modèle qui a longtemps dominé la conception occidentale de la religion, axée sur la croyance. Son sens critique, cependant, et, à l'opposé, les failles de son système rationnel du croire fragilisent ce modèle et le livrent à la discussion, s'il est mis à l'épreuve de la comparaison avec d'autres régimes que celui du monothéisme des religions du Livre.

\section{La crédibilité objective de la foi chrétienne chez Origène}

Lorsque Celse définit les chrétiens par la convention qui est censée les unir, il assimile leur sunthêma ("signe d'alliance, convention») à leur pistis ( «foi »): «Leur convention, dit Celse cité par Origène, est d'autant plus étonnante qu'on peut mieux prouver qu'elle ne repose sur aucun fondement solide. Elle n'a de fondement solide que la révolte, l'avantage qu'on en espère et la crainte de ceux du dehors : telle est l'assise de leur foi » (Contre Celse, III,14, 1. 1-5 ${ }^{3}$ ). Rébellion, dissensions internes, rivalités, autant de défauts qui excluent cette religion de la société ambiante et qui en outre réduisent à néant son assise, limitée, bizarrement pour le philosophe grec, à une « foi ». Lorsque Celse reprend le mot convention, c'est encore pour

3. Une édition et traduction française annotée du Contre Celse par M. Borret est parue dans Sources chrétiennes, 132 (1967); 136 (1968); 147 (1969); 150 (1969); 227 (1976). 
fustiger une religion qui évite les signes visibles de la présence dans la cité et la participation aux cultes publics, et qui se replie dans le secret: "Ensuite, écrit Origène, il déclare que nous évitons d'édifier des autels, des statues et des temples; car il croit que c'est la convention qui garantit notre association secrète et mystérieuse " (VIII,17, 1. 1-3). La réponse d'Origène, quelques décennies plus tard, consiste à affermir le fondement de cette «convention" qui unit les adeptes de sa religion, en donnant pour origine de la «foi » l'enseignement de Dieu, par les prophètes, et pour cause du développement de la doctrine "l'apparition sensible de Dieu» (III,14, 1. 520). Il affirme que la «foi» des chrétiens repose tout entière sur Dieu et sur les promesses du Christ aux justes et ses enseignements sur le châtiment des injustes (VIII,51, 1. 11-14). On revient toujours à la foi. Origène perçoit le pamphlet de Celse comme «écrit contre les chrétiens et leur foi» (II,69, 1. 28-29). Ceux qui ne sont pas chrétiens sont qualifiés globalement d' «étrangers à la foi » (voir par ex. I,51, 1. 18; V,50, 1. 8; VII,8, 1. 13; VIII,73, 1. 14).

Une grande partie de la réplique d'Origène développe le débat sur les raisons de croire en la validité de cette "foi ", en faisant appel d'une part à l'autorité des traditions et des documents, d'autre part aux preuves tirées de l'histoire. La question de la croyance, dans ses modalités diverses, y est centrale. L'effort est de rendre plausible, par des arguments rationnels, l'adhésion à cette « foi », de transformer ce qui fait de la « foi » une opinion individuelle et indécise, soumise à l'inconstance de l'affectivité, en une conviction rendue digne d'être partagée par les témoignages trouvés dans les concordances entre, d'un côté, les récits hérités et les événements qui sont la matière du croire et, de l'autre, l'examen des faits, en l'occurrence de l'expansion du christianisme. La querelle oppose aux traditions religieuses et aux mythes dont Celse affirme la supériorité, les récits et prophéties que les chrétiens se sont appropriés ainsi que les leçons de l'histoire récente et contemporaine. Les interrogations sur la crédibilité des preuves invoquées de part et d'autre rencontrent la question de la vérité historique, tout en cantonnant la réflexion sur le terrain de la vraisemblance, plus ou moins grande, qui est celui de la rhétorique, et non de la démonstration scientifique. Ainsi Origène reproche-t-il à Celse d'avoir cru «aux histoires des barbares et des Grecs sur l'antiquité des peuples qu'il mentionne, tandis qu'il accuse de mensonge les histoires de ce seul peuple» des Juifs (Contre Celse, I,14, 1. 6-9). Celse aurait dû citer les textes mêmes s'il voulait «détourner ceux qui sont attirés, à son avis, par leur caractère persuasif [pithanotês] de l'adhé- 
sion aux prophéties et de la foi, fondée sur elles, en Jésus comme au Christ » (IV,2, 1. 18-23). Le "caractère persuasif ", la pithanotês, renvoie aux seuls raisonnements plausibles, toujours suspects d'être spécieux, illusoires et trompeurs, de l'art rhétorique et non pas à la démonstration logique, capable de produire un savoir, une science. De même Origène ramène-t-il au rang de simples arguments persuasifs, pithana, les preuves alléguées par Celse (V,1, 1. 20) ou lui relance le grief opposé aux chrétiens: «... à moins, par hasard, que lui qui se moque de ceux qui professent une foi simple [psilê pistis] ait voulu lui-même que nous ajoutions foi à ce qu'il avançait, bien qu'il ait prétendu non pas exprimer son avis, mais enseigner» (IV,54, 1. 4245 ; voir I,9). On n'est pas loin de l'accusation de faux témoignage, qu'Origène n'hésite pas à formuler ailleurs (VI,38, 1. 30). Le débat en reste au niveau de la «persuasion» obtenue par l'éloquence judiciaire, qui produit une croyance qui peut être fausse, comme Socrate, selon Platon, le faisait reconnaître à Gorgias ${ }^{4}$. Et quand la discussion prétend évaluer la rationalité des arguments, elle dénonce chez l'adversaire les procédés de l'éristique; c'est le cas à propos du refus de Celse d'accepter en faveur du Christ les preuves par la prédiction des prophètes; Origène reconstruit sa critique en y dénonçant un vice de raisonnement, un paralogisme qui prive l'argumentation de toute efficacité apodictique :

Si les vrais prophètes du Dieu suprême disent que Dieu subira l'esclavage, la maladie ou même la mort, ces malheurs arriveront à Dieu, puisque les prophètes du grand Dieu disent nécessairement la vérité. D'autre part, si les vrais prophètes du Dieu suprême disent ces mêmes choses, puisque ce qui est par nature impossible ne peut être vrai, ce que les prophètes annoncent de Dieu ne saurait arriver. Or, quand deux prémisses ont des conséquences contradictoires en raison de ce qu'on appelle le syllogisme des deux propositions, l'antécédent des deux prémisses est détruit, en l'espèce: les prophètes prédisent que le grand Dieu va subir l'esclavage, la maladie, la mort. La conclusion est donc que les prophètes n'ont pas prédit que le grand Dieu subirait l'esclavage, la maladie, la mort ${ }^{5}$. (VII,15, 1. 9-21; voir VII,14, 1. 17-27)

4. Gorgias, 454 c-455 a ; voir Théétète, 200 e-201 c ; Politique, 304 c.

5. Origène donne ensuite la forme du paralogisme : si le premier, le second; si le premier, non le second; donc non le premier, et il cite l'exemple que les stoïciens proposent en la matière (VII,15, 1. 21-35). Il s'agit là du « syllogisme des deux conditionnelles ", dont Origène donne l'analyse la plus complète, comme l'a montré J.M. Rist (1981, 73-75). 
À cette réduction à l'absurde, il ajoute que les prophéties n'ont rien de semblable à l'hypothèse retenue par Celse: elles n'ont pas prédit que Dieu serait crucifié, mais «l'homme que l'intelligence perçoit en Jésus» (VII,16, 1. 19). Proposition, au demeurant, qui eût fort irrité les tenants d'une formule qui eut son heure de gloire et son odeur de sainteté au vi ${ }^{\mathrm{e}}$ siècle, sous Justinien: "Un de la Trinité a été crucifié. »

Ainsi, d'un point de vue grec, qui est aussi celui d'Origène, la critique et la défense de la «foi » chrétienne se situent au niveau de la persuasion rhétorique propre à la controverse, sans se hisser jusqu'à la réflexion capable de produire un savoir assuré; en dialectique, elles ne dépassent pas l'exigence d'exactitude et de rigueur à attendre de la méthode par laquelle le questionneur met à l'épreuve la thèse de l'adversaire et au moyen de laquelle le répondant se rend capable de mettre en échec le questionneur éristique en examinant la forme de ses raisonnements, la méthode dont Aristote se considère comme l'inventeur à la fin des Réfutations sophistiques (chap. 34, voir Dorion 2002); il ne s'agit pas de la dialectique au sens platonicien du terme, comme méthode d'ascension vers le bien véritable.

La conviction qu'Origène s'efforce de faire naître, contre les griefs d'inanité et de fausseté formulés par Celse, à propos de la crédibilité de la «foi » chrétienne, est que celle-ci est préférable aux autres croyances religieuses, qu'elle est plus conforme au modèle de vie parfaite et que sa déraison même est le signe d'une sagesse supérieure. Il affronte aussi la question du crédit qu'il est possible d'accorder aux récits évangéliques, par exemple à l'apparition de la colombe au-dessus de Jésus au moment de son baptême. Pour répondre à l'objection prêtée par Celse à un Juif, il commence par une considération générale, qui concerne toute " histoire, fût-elle vraie» : "vouloir établir qu'elle a eu lieu et en donner une représentation compréhensive est une chose des plus difficile et, dans certains cas, impossible» $(\mathrm{I}, 42)$. Cette considération s'appuie sur des exemples empruntés aux récits concernant Achille à Troie, Edipe ou encore les Héraclides; elle a retenu l'attention de Paul Veyne (voir 1983, 70) : les réflexions d'Origène, qui invite « tout lecteur judicieux de ces histoires » à discerner « d'une part ce qui mérite son adhésion et ce qu'il interprétera allégoriquement en recherchant l'intention de ceux qui ont forgé de telles fictions, et d'autre part ce qu'il refusera de croire, comme écrit pour procurer quelque agrément ${ }^{6} »(\mathrm{I}, 42)$, indiqueraient que,

6. Ou (le texte est douteux) : «par complaisance pour certains ». 
pour les Grecs, une tradition mythique est vraie malgré le merveilleux; la démonstration de l'historicité des événements rapportés est en effet rendue malaisée, selon Origène, par «la fiction qui s'entrelace avec l'opinion qui prévaut chez tous que la guerre à Ilion entre Grecs et Troyens a réellement eu lieu» (ibid.). Ce n'est pas cependant d'un tel témoignage que l'on peut conclure que, aux yeux des Grecs, une science du mythe "ne se proposerait pas de faire comprendre l'erreur, mais d'apprendre à s'en garder» (Veyne 1983, 71). L'erreur, pour Origène, et pour les allégoristes auxquels il se rallie, ne serait pas seulement de croire naïvement en la réalité des éléments de la fiction, mais aussi, et peut-être surtout, de ne pas croire en l'existence d'un sens caché sous les invraisemblances du récit, une fois qu'on a fait la part des traits qui sont là pour le plaisir du lecteur. La remarque qui conclut cette considération générale d'Origène est en effet capitale: son dessein était «d'établir que les lecteurs ont besoin d'un jugement sain et d'un examen approfondi, et en quelque sorte d'entrer dans l'intention des écrivains, pour trouver dans quel esprit chaque événement est décrit» (I,42). La conclusion fait le lien entre l'interprétation des «histoires " composées par les Grecs et le commentaire de l'épisode du baptême de Jésus rapporté dans l'Évangile. Sur le plan de la théorie herméneutique, la relation est fortement présente entre les récits des Grecs et les Écritures: c'est le même argument de «l'impossibilité » $(\mathrm{I}, 42,1.6)$ qui ici disqualifie la légende comme document digne de foi, en orientant la lecture vers l'allégorisation, et qu'Origène avait développé dans le traité d'herméneutique au livre IV du traité Des Principes pour repérer dans la trame historico-législative des Écritures les signes de la présence d'un sens second, plus profond. Le tri opéré dans ce passage du Contre Celse distingue donc deux formes du croire, celui de l'historien qui critique ses sources et pense pouvoir atteindre à travers elles les faits, la réalité événementielle, et celui de l'interprète qu'une méthode apprise et une théorie du mythe ont convaincu que la légende recèle un enseignement de portée générale, moral, physique et/ou métaphysique, ou encore religieux, selon une visée philosophique. À ces deux croyances différentes, inculquées par l'apprentissage et la pratique de métiers particuliers pourvus de leurs technai, s'en ajoute chez Origène une troisième: la foi en l'inspiration divine des Écritures, dont l'auteur véritable serait l'Esprit saint qui use du truchement des prophètes, des évangélistes et des apôtres. Il convient cependant de remarquer les analogies entre cette foi et les convictions des philosophes allégoristes, pour lesquels c'est la nature ellemême qui aime à se cacher dans le langage et dans les mythes des traditions 
ancestrales ${ }^{7}$. Dans le domaine proprement religieux, les débats du livre VII du Contre Celse, où Origène oppose les prophéties bibliques aux oracles des Grecs, seraient à étudier pour repérer l'appartenance de l'adhésion aux uns et aux autres à un registre commun, et pour voir dans quel sens le chrétien s'efforce d'opérer la différenciation. Retenons simplement deux traits qu'Origène invoque pour établir l'authenticité divine de l'inspiration prophétique: celle-ci est illuminatrice, exempte de la possession qui égare et aliène l'intelligence; elle est accordée à des prophètes dont la vie exemplaire est connue et elle a des effets moralement bienfaisants. La défense de la foi en leur inspiration ramène au niveau de la persuasion propre à la controverse, tout en maniant un argument polémique présenté comme fondé sur une certitude: les inspirateurs des oracles païens sont des démons malfaisants. En ce qu'il oppose croyance à croyance, pour mettre en évidence la dignité plus haute de celle des Juifs et des chrétiens, l'argument est plus efficace que le recours, un moment envisagé, puis congédié par feinte complaisance, aux critiques formulées par des philosophes grecs, péripatéticiens et épicuriens, contre la foi donnée aux oracles.

\section{La distinction entre la pistis et la croyance}

La foi en Dieu et en Christ, dont la plausibilité est fondée par Origène sur les faits de l'histoire (contre les négations de Celse) et sur la valeur morale de ses effets, est magnifiée aussi par l'opposition sémantique entre la pistis, le pisteuein, et l'expression theous nomizein. Wilhelm Fahr (1969) a pu montrer que celle-ci, qui n'apparaît pas avant les Perses d'Eschyle, en 472, porte en germe la possibilité de l'athéisme. À l'époque ancienne, le concept de foi-croyance est absent de la langue grecque et avant les sophistes, la possibilité de l'incroyance, qui va de pair avec la croyance, n'est pas imaginable. S'il y a foi, elle s'exprime par le culte. Il n'y a pas de foi comme simple croyance-opinion. Le verbe nomizein a eu très tôt une double acception: l'opinion, non contraignante, opposée au savoir, et la pratique conforme, obligatoire, qui engage, par référence au nomos ( «coutume» ou «loi »). Une incertitude accompagne les emplois du terme: ceux qui ont une opinion ont-ils raison de l'avoir ? est-elle vraie ou fausse? ceux qui ont une coutume

7. Pierre Hadot rappelle le lien entre «physiologie» (discours sur la nature) et «théologie » chez les auteurs qui, du $\mathrm{I}^{\mathrm{er}}$ au $\mathrm{IV}^{\mathrm{e}}$ siècles de notre ère, ont donné à l'aphorisme d'Héraclite le sens «La nature aime à se cacher» en l'associant à la métaphore des «secrets de la nature» $(2004,55-60)$. 
agissent-ils de façon correcte? Cette indétermination entraîne les critiques des philosophes présocratiques sur la tradition, les coutumes et l'opinion de la foule. Le tour theous nomizein, qui se diffuse largement chez Hérodote et se retrouve plus tard chez Platon, a une dualité de sens parallèle: honorer les dieux selon l'usage, et tenir l'existence des dieux pour réelle, avec la différence que, dans le cas de cette expression nouvelle, le second sens est postérieur au premier. C'est avec Protagoras qu'apparait l'idée de l'inexistence possible des dieux, qui prend une forme plus radicale chez Diagoras de Mélos. L'expression du doute est liée à la critique du nomos ( «loi »), opposé à la phusis («nature»).

Origène joue sur le double sens du tour pour rejeter le polythéisme et lui opposer la foi chrétienne. Il développe la réplique provocante de Justin: « Nous reconnaissons être les athées des prétendus dieux de ce genre» (Apologie, I,6,1). Ainsi reproche-t-il à Celse d'avoir délibérément passé sous silence le mythe «des dieux supposés [nomizomenoi theoi] », aux passions tout humaines, enseigné principalement dans les écrits d'Orphée (Contre Celse, I,17; voir VI,54, 1. 17). La référence à l'usage, la coutume cultuelle, reste vivante, à côté du second sens, dans le passage où il proteste contre le jugement porté par Celse sur les prophètes du peuple des Juifs:

Les nations dont ils étaient environnés croyaient [episteue] chacune selon ses traditions ancestrales recevoir des oracles et des divinations des dieux qu'elles vénéraient [nomizomenoi theoi]; eux seuls, au contraire, qui avaient été éduqués dans le mépris de tous ceux qui passaient pour des dieux [nomizomenoi theoi] chez les nations et y voyaient non pas des dieux mais des démons — puisque leurs prophètes disaient: Tous les dieux des nations sont des démons (Ps 95,5) - n'auraient eu personne qui fît profession de prédire et fût capable de retenir ceux qui, par désir de prévision des événements futurs, voulaient s'en aller vers les démons des autres nations ${ }^{8}$ ?

La même ambiguïté polémique apparaît dans l'expression hê peri tôn nomizomenôn theôn hupolêpsis ( "l'opinion [qu'ont les païens] des prétendus dieux » ou: «des dieux du culte»). Le participe parfait passif du verbe

8. Contre Celse, III,2, 1. 22-30; voir VII,69, 1. 21-22; VII,48, 1. 16; VIII,38, 12. En $\mathrm{V}, 29,1.5$ et VIII,30, 1. 15, c'est l'expression « ceux qui sont dits dieux [hoi legomenoi theoi] » qui est employée de la même façon.

9. Contre Celse, VIII,38, 1. 12. L'hupolêpsis ( "opinion ») s'oppose couramment à la pistis ( "foi ") comme le verbe hupolambanein à pisteuein (voir Origène, Commentaire sur Matthieu, X,17 [Benz et Klostermann 1937, p. 21, 1. 20-21] ; X,17 [p. 27, 1. 30]; XII,9 [p. 80, 1. 19]). 
nomizein est moins malléable; il retient mieux la notion de coutume, d'usage traditionnel, de pratique rituelle: "Même ceux qui, d'après vous, sont prêtres de certaines statues et gardiens des temples de ceux que vous tenez pour dieux, ont soin de garder leur main droite sans souillure pour les sacrifices, afin d'offrir à ceux que vous dites dieux les sacrifices en usage [nenomismenai thusiai] avec des mains pures de sang et de meurtre» (Contre Celse, VIII,73, 1. 17). En général, le verbe nomizein est employé pour désigner un simple avis, une opinion non plausible ou fausse, une illusion, un faux-semblant, voire une imposture, comme le montrent plusieurs passages des tomes du Commentaire sur Matthieu conservés en $\operatorname{grec}^{10}$. La forme nomisteon signifie «il faut estimer/considérer/penser» et non pas «il faut croire» au sens fort ${ }^{11}$. Les "usages» (ta nomizomena), d'autre part, sont à distinguer de l'acte fondateur de la loi et peuvent ne pas être bons. Clément d'Alexandrie avait répété la précaution des défenseurs du nomos consistant à séparer le nom du verbe nomizein, qu'on trouve dans le dialogue pseudo-platonicien Minos $^{12}$. En matière de vénération religieuse et de foi en Dieu, Origène prend une autre direction, en tirant une conclusion de type philosophique de l'usage polémique opposant le theous nomizein des Grecs à la piété véritable à ses yeux des Juifs et des chrétiens. À Celse qui considère qu' "il y aurait impiété à enfreindre les usages établis dès l'origine dans chaque région ", en particulier parce que « les différentes parties de la terre ont été dès l'origine attribuées à différents surveillants » $(\mathrm{V}, 25,1.9-14)$ tutélaires et réparties en autant de gouvernements, il oppose, en reprenant les exemples classiques de la polémique contre la multiplicité des codes et des pratiques religieuses, la diversité et l'immoralité des coutumes traditionnelles qui sont censées avoir été édictées par ces puissances. Il en conclut ironiquement: d'après Celse, « la piété serait divine non point par nature mais par quelque convention et par coutume [ou phusei...alla tini thesei kai nomisei] » (V,25, 1. 9-14; V,27, 1. 1-19, 22-23). Le contexte permet de dire que pour Origène, au contraire, la religion des Juifs (et par conséquent des chrétiens) est réellement divine parce qu'elle leur donne pour unique objet de vénération le Créateur

10. XIII,20 (Benz et Klostermann 1937, p. 235, 1. 3) ; X,20 (p. 27, 1. 13) ; XVII,29 (p. 667, 1. 31); XVII,12 (p. 615, 1. 17); XI,12 (p. 53, 1. 12); XIII,16 (p. 222, 1. 5); XI,15 (p. 59, 1. 7); XIII,22 (p. 240, 1. 11); XVI,22 (p. 551, 1. 13).

11. Commentaire sur Matthieu, X, 12 (Benz et Klostermann 1937, p. 13, 1. 21), etc.

12. «La loi n'est pas ce qui est légalement en usage (car ce qui est vu n'est pas la vision), ni toute décision (ce n'est pas la mauvaise), mais la loi est décision bonne » (Stromates, I,25,166,4 ; voir Minos, 313 c-315 a). 
de l'univers, dont la providence a assigné aux nations des anges tutélaires. C'est en ce sens, et selon un raisonnement circulaire, que la foi monothéiste est «par nature » et non "par convention et par coutume». Le mot rare nomisis vient aggraver l'opposition entre thesei et phusei, qui avait divisé aussi les philosophes et continuait de le faire au temps d'Origène à propos de l'origine du langage : la "convention » renvoie au moins à l'activité raisonnée d'un nomothète, qu'il soit divin ou humain; la nomisis associe à la coutume la fragilité de l'opinion et recueille ainsi tout l'héritage des impiétés passées et des contestations antireligieuses. Prétendre fonder la foi sur la «nature ", c'est parachever l'apologie qui la présente comme la plus crédible, en la dotant de la force de la nécessité. Une telle légitimation est en accord avec le propos de démontrer que les doctrines mêmes de la foi chrétienne sont absolument en accord avec les notions communes (voir III,40; VII,52) et avec la thèse selon laquelle le chrétien est le vrai philosophe, en ce qu'il s'élève au-dessus de la superstition, de la deisidaimonia, parce qu'il préfère la vénération du Dieu de l'univers au respect des traditions ancestrales $(\mathrm{V}, 35)$. En des termes voisins, Origène peut distinguer de façon générale deux lois :

... l'une, la loi de la nature, dont on peut dire que Dieu l'a instituée, l'autre, la loi écrite des cités. [...] Quand la loi de la nature, c'est-à-dire de Dieu, ordonne le contraire de la loi écrite, vois si la raison n'impose pas de congédier les textes et l'intention des législateurs, pour se donner au Dieu législateur et choisir une vie conforme à son Logos...

Et il ajoute: «S'il est raisonnable de préférer sur les autres points la loi de la nature, qui est la loi de Dieu, à celle qui est écrite et promulguée par les hommes en contradiction avec la loi de Dieu, combien plus ne le serat-il pas quand il s'agit de lois sur le culte à rendre à Dieu ${ }^{13}$ ? » (V,37, 1. 1-9 et 1. 18-22)

13. Dans tout ce développement, Origène insiste sur le caractère raisonnable de la démarche des chrétiens : ce sont eux les vrais philosophes, et non ceux qui, pour respecter les usages ancestraux, resteraient prisonniers de la superstition (deisidaimonia); ce sont eux qui adhèrent à ce que la raison prescrit, eux dont la conviction se fonde sur le choix de la raison; l'assentiment qu'ils donnent à leur croyance est le produit d'une détermination réfléchie. Une citation assez longue est ici nécessaire :

Car si c'est à cause de la philosophie et des leçons proscrivant la superstition qu'ils peuvent, au mépris des traditions, manger des aliments interdits depuis le temps de leurs ancêtres, pourquoi les chrétiens ne le pourraient-ils pas? La raison impose [logou hairoûntos] en effet de ne pas s'arrêter aux statues, aux images ou même 
Philosophie véritable, institution naturelle et divine, enseignement d'Écritures qui entrelacent aux témoignages de l'histoire des leçons inspirées et mystérieuses, source de vie dont la puissance est attestée par la rapidité de sa diffusion, autant de définitions origéniennes de la foi chrétienne qui la présentent comme la meilleure des croyances, et, plus encore, comme le fondement de la seule religion réellement pieuse. Cette description en fait l'objet parfait, supérieur aux outrages et offert à l'admiration universelle. Elle ne répond cependant que partiellement à la critique de Celse, qui porte aussi sur les modalités de l'adhésion subjective au contenu de la foi. En philosophe, Celse exige qu'on suive la raison et un guide raisonnable pour pouvoir donner sans risque son assentiment à des doctrines. Sinon, on court le danger d'être dupé par des charlatans pervers, par certains chrétiens qui, "ne voulant pas même donner ni recevoir de raison sur ce qu'ils croient [pisteuousi], usent de ces paroles : "N'examine pas, mais crois; la foi te sauvera" [...] "La sagesse

aux créatures de Dieu, mais de les dépasser et de présenter leur âme au Créateur : pourquoi, se conduisant comme les philosophes, ne seraient-ils point irréprochables? Si pour sauver sa thèse, Celse, avec ses adeptes, affirme que même un philosophe devra observer les traditions ancestrales [ta patria], alors les philosophes deviendront parfaitement ridicules, par exemple parmi les Égyptiens en se gardant de manger de l'oignon pour observer les traditions [...]. Si l'un d'eux devenu philosophe gardait les traditions, ce serait un philosophe ridicule, sans philosophie dans sa conduite. Il en va de même quand on a été conduit par la raison [hupo tổ logou] à adorer le Dieu de l'univers si, à cause des traditions, on reste abaissé devant les images et les statues humaines; et si on refuse de s'élever par détermination réfléchie [proairesei] jusqu'au Créateur, on est semblable alors à des gens qui, malgré les leçons de la philosophie, craindraient ce qui n'est pas à craindre et jugeraient impie de manger de tels mets. (V,35, 1. 9-33)

Le terme logos ne désigne pas ici le Logos divin (selon la traduction de M. Borret), mais la raison humaine (voir V,37, 1. 7). Dans un contexte semblable, à propos de la foi dans le Créateur, Origène a de nouveau recours à la notion de détermination volontaire (proairesis), en VIII,53 (voir VI,57, 1. 33-36). Clément d'Alexandrie avait donné à cette notion un rôle important dans la définition de la pistis, notamment en Stromates, II, 2,9, sous l'influence d'un modèle principalement aristotélicien selon J. Lössl (2002). La même insistance que dans le Contre Celse sur le libre choix raisonnable apparaît dans une définition de la pistis transmise par un fragment caténal sur le Ps 115,1 (Patrologia Groeca, 12, 1576C) qu'il faut restituer à Évagre : «La foi est un consentement raisonnable de l'âme douée du libre arbitre » (voir Guillaumont 2004, 206, note 1 ).

Guy G. Stroumsa (1998) a retenu le tour individuel de cette conviction pour mettre en évidence l'innovation qu'elle implique dans la façon de concevoir la religion, par rapport au traditionalisme et au relativisme de Celse. 
en ce monde est un mal, et la folie un bien"14 » (I,9, 1. 1-14). Pour relever le défi, Origène est amené à reprendre sa distinction si fréquente entre les simples, les progressants et ceux qui aspirent à la perfection. Corrélativement, il existe plusieurs degrés dans la foi. Il peut même y avoir des erreurs, des façons de croire et des contenus de croyance faux. Dans ce cas, c'est encore le verbe nomizein qu'Origène emploie pour désigner de telles méprises. Il rejette par exemple dans son Commentaire sur Matthieu une conception qu'il juge grossière des effets de la réception du pain eucharistié: «...ce n'est pas ce qui entre dans la bouche qui sanctifie l'homme, même si les gens frustes croient [nomizein] que ce que l'on appelle le pain du Seigneur les sanctifie» (XI,14). La croyance en l'efficacité du rite doit être complétée par une doctrine, qu'Origène expose ensuite, pour répondre autant à sa propre exigence de rationalité ${ }^{15}$ qu'à celle de ses lecteurs, l'explication recherchée tenant compte de la distinction entre la matière du pain et la parole prononcée sur lui, des dispositions du récepteur et du réseau de textes scripturaires composé autour du rite par celui qui tente d'élucider le contenu de la foi. De même, dans le Contre Celse, il reprend l'examen de la foi en la résurrection des corps, en se fondant sur le discours (scripturaire) «qui enseigne longuement la différence entre le corps pour ainsi dire semé et celui qui en est comme ressuscité » $(\mathrm{V}, 19,1.6-8)$, et il reproche à Celse d'être «incapable de juger qu'il ne faut pas penser [nomizein] que le sens voulu par ces auteurs savants [c'est-à-dire les écrivains inspirés des textes sacrés] soit présenté par ceux qui ne professent rien de plus que la foi relativement à la doctrine des chrétiens » (20, 1. 6-9). Le mot pistis désigne ici la « foi » des simples, qui croient en la résurrection de la chair sans s'interroger sur sa nature ou sans voir les difficultés que comporte la croyance en l'identité du corps terrestre et du corps ressuscité.

Origène revendique cependant le droit pour les chrétiens d'admettre cette foi simple «comme utile à la foule » et "d'enseigner à croire même sans réflexion à ceux qui ne peuvent tout laisser et poursuivre l'examen de la doctrine » (I,10, 1. 2-5). Il transpose dans le christianisme la distinction antique entre vie active et vie contemplative, réservant la seconde au petit nombre capable, dans le loisir ou par l'ascèse, de se déprendre des affaires

14. Celse, cité par Origène, exploite ici des passages évangéliques, puis adapte à son propos 1Co 1,18-21 et 3,18-19 (voir Perrone 1998, 247).

15. On sait qu'Origène s'efforce constamment de remplir le programme indiqué dans le Traité des principes, IV,1,1: «Nous tentons d'affermir notre foi par la raison ». 
quotidiennes ${ }^{16}$. Quant à la « folie » de la foi, elle est un bien par rapport aux opinions de la fausse sagesse ${ }^{17}$ et elle n'est pas « folie tout court », mais « folie de la prédication » $(\mathrm{I}, 13,1.34-35)$, «Jésus Christ proclamé crucifié ${ }^{18}$ » (1. 35, 38). Cette prédication est présentée comme le remède voulu par Dieu et destiné à "sauver les croyants », puisque Dieu n'a pas été connu «dans la sagesse de Dieu ", selon 1Co 1,21; c'est en raison des circonstances que le Logos a voulu aussi " la foi simple ", " pour ne pas laisser les hommes entièrement désemparés» (1. 25-27). Néanmoins, "au sentiment du Logos, il vaut bien mieux donner son assentiment aux doctrines [sugkatatithesthai tois dogmasi] avec réflexion et sagesse qu'avec la foi simple» (1.23-25). Ce n'est pas le lieu de commenter ces assertions d'Origène, mais de discerner ce que son jugement nous apprend sur la forme de croyance de la majorité des chrétiens de son temps, qui sont précisément ceux qu'il appelle "les simples », comme l'a montré Hällström (1984). Un passage de peu antérieur apporte un éclairage utile. Origène s'y moque de Celse, qui tiendrait tout son savoir sur les chrétiens des plus ignorants d'entre eux, sans s'être instruit auprès des gens compétents du sens des Écritures. C'est l'occasion pour lui d'énoncer une loi générale, qui vaut pour tous les peuples détenteurs de livres et de rites sacrés, Égyptiens, Perses, Syriens, Indiens: les sages du pays interprètent rationnellement les mystères, alors que la foule les accomplit au niveau de symboles (I,12, 1.25-37). L'adverbe sumbolikôs veut dire ici que les simples adhèrent à la surface des rites, accordent toute leur foi aux formes littérale des textes et matérielle du culte. Si l'on fait abstraction du jugement de valeur ainsi exprimé, on peut retenir seulement la conception de la religion qui domine l'apologie d'Origène: textes prescriptifs et codes cérémoniels sont les «symboles » d'un sens à découvrir par un travail de la raison, mené par des experts dont le genre de vie est adapté à cette mission et qui sont pour les autres des maîtres de sagesse conduisant à un savoir, consistant en un corps de doctrines, et en même temps à une perfection spirituelle. L'ensemble des «symboles", pris en eux-mêmes, a cependant sa suffisance propre. Le christianisme, à cet égard, est semblable aux autres religions, telles qu'elles sont perçues par les philosophes d'obédience platonicienne à cette époque.

16. La distinction est expressément formulée dès la première réponse au grief de crédulité retenu par Celse $(\mathrm{I}, 9)$.

17. Origène a cité 1 Co 3,18-19.

18. Origène cite 1Co 1,23-24. Il affirme ailleurs que Dieu agrée aussi bien la foi des simples que la piété accompagnée de raison de ceux qui ont plus d'intelligence (Contre Celse, VII,46, 1. 6-7). 
Il n'en diffère, dans l'intérêt de la foule, selon Origène, que par l'excellence de ses commandements, par la qualité de ses rites, par la justesse de ses articles de foi, qui assurent les uns et les autres la cohésion des communautés et convertissent les adhérents, par la prédication du Christ et par l'adoration du Dieu créateur, dispensateur de tous les biens, à une conduite méritant d'être récompensée dans l'au-delà. Souvent, en effet, Origène insiste sur l'importance de la doctrine de la rétribution pour le salut des «simples».

\section{La pistis chez Origène}

En termes chrétiens, pauliniens, la condescendance se teinte d'estime quand l'échelle des degrés d'initiation devient une hiérarchie des "charismes": "Ainsi, la sagesse divine, qui diffère de la foi, est le premier de ce qu'on appelle les charismes de Dieu. Après elle le second, aux yeux de ceux qui ont une science précise en ce domaine, est ce qu'on appelle la connaissance. Et le troisième est la foi, puisqu'il faut que soient sauvés même les plus simples qui s'adonnent de leur mieux à la piété " (Contre Celse, VI,13, 1. 23-27; suit la citation de 1Co 12,8-9). La source des charismes étant unique, la puissance de Dieu (voir V,1, 1. 13-36; I,62, 1. 21-63), il y a continuité entre eux, et la «sagesse divine» est le déploiement ultime de la «foi». Quand Origène s'interroge sur la signification de sa propre foi, sans doute marquet-il les différences entre les degrés de compréhension. Ainsi, lorsque, partant de la foi en la réalité des apparitions du Christ après sa résurrection, à partir d'Ac 1,3, Jn 20,26, et surtout 1Co 15,3-8, il s'exclame: "Qu'elles me paraissent grandes, admirables, sans proportion avec le mérite non seulement de la foule des croyants, mais encore de l'élite en progrès dans la doctrine, les vérités de ce que contient ce passage! Elles pourraient montrer la raison pour laquelle, après sa résurrection d'entre les morts, il n'apparaît point de la même façon qu'auparavant » (II,63, 1. 22-27). La foi cependant est de l'ordre de la vérité. Le modèle épistémologique de Platon est certainement à l'arrière-plan de la réflexion d'Origène sur les degrés de la connaissance religieuse, comme le suggère, dans le Contre Celse lui-même, la comparaison esquissée entre la théorie platonicienne de l'intellection et les voies de la contemplation menant le philosophe chrétien des réalités invisibles au Dieu éternel (voir VII,46), mais les liens entre les modes de perception sont plus forts chez lui. L'allégorie de la ligne dans la République, VI, 509 d 6-511 e 5, laisse la pistis, même si elle est supérieure à la conjecture (eikasia) du côté de la doxa, qui a pour objet le devenir. La «foi » est le corrélat cognitif des réalités sensibles visibles. Certes, à ce titre, elle 
apparaît au même plan que la «foi » selon Origène, qui s'applique à la lettre et aux éléments matériels. Mais elle est, selon le Timée, séparée de la vérité: "Ce que l'être est au devenir, la vérité l'est à la foi » (29 b-c). À l'intellection "seuls participent les dieux, et une toute petite catégorie d'êtres humains" (Timée, $51 \mathrm{e}$ ), de même que très peu ont part au charisme de «la sagesse divine ». Mais contrairement à la foi qui, selon Platon, peut être ébranlée par la «persuasion» et n'est pas produite par l'enseignement (voir Timée, 51 d-e; Gorgias, 454 c-455 a Théétète, 200 e-201 c), la foi authentique, selon Origène, ne peut être modifiée, parce qu'elle est le fruit d'une doctrine et d'une prédication, celles de Jésus, qui n'ont pas consisté «en discours persuasifs de la sagesse qui use du style et de la composition » rhétorique, mais en démonstration de la puissance de Dieu ${ }^{19}$. Si la « foi » se trouve ainsi pourvue d'une stabilité qui était l'apanage de l'«intellection » pour Platon, la «sensation ", qui était le mode cognitif propre à la «foi » platonicienne, est élevée au rang de faculté de connaissance la plus haute, en tant que "sens divin ", selon le contenu que donne Origène à la parole de Pr 2,5 (selon la Septante): «Tu trouveras un sens divin », appliquée aux prophètes; ceux-ci « regardaient divinement, écoutaient divinement, goûtaient et sentaient de même façon, pour ainsi dire d'un sens qui n'est pas sensible; et ils touchaient le Logos avec foi, si bien qu'une émanation leur arrivait de lui pour les soigner ${ }^{20}{ }^{\prime}$. Il est remarquable que, dans ce passage, Origène fonde sa théorie sur une induction partant d'une croyance générale (pepisteutai), concernant les représentations de réalités "divines" ou d'événements à venir produites en songe: il n'est pas absurde, selon lui, que des impressions semblables, qui ne font pas intervenir les sens corporels, affectent aussi «la partie directrice » de l'âme, l'intellect, à l'état de veille, par le genre de perception qui est "le sens divin ». L'explication théologique rapporte ces impressions aux effets d'un charisme spirituel, dont bénéficient aussi les maîtres qui dispensent un enseignement "à ceux qui ont une connaissance approfondie du christianisme", et se fonde sur l'autorité de Paul (1Co 12,8) (Contre Celse, III,18, 1. 5-9). Elle évoque en même temps une expérience, sous la forme d'un "contact» (haphên): dans le cas des prophètes, «le contact, pour ainsi dire, de ce qu'on appelle l'Esprit Saint avec leur âme rendait leur intelligence plus perspicace, leur âme plus limpide»(VII,4, 1. 5-8).

19. Contre Celse, I,62, 1. 20-63, citant 1Co 2,4-5; Ps 67,12-13; 147,4; voir Contre Celse, III,39.

20. Contre Celse, I,48, 1. 39-44, se référant aussi à 1Jn 1,1; voir Rahner 1932. 
Cette expérience est accordée à ceux qui lisent les Écritures comme il convient, c'est-à-dire avec la foi en leur inspiration : ils sont alors remplis d'une ardeur divine qui fait jaillir dans leur âme une lumière supérieure à la flamme dont parle Platon dans la Lettre VII (voir Contre Celse, VI,4-5). Origène va jusqu'à appliquer à son travail personnel d'interprétation des Écritures et à la longue recherche du sens la perception de ce contact: "... nous ne renoncerons pas, en touchant à tâtons les paroles de vie, à tenter de recevoir d'elles la puissance qui s'écoule sur celui qui les touche avec foi» (Commentaire sur Jean, X, 28, 172 ; voir Harl 1975 ; 1983, 155). En deçà donc, ou au-delà du discours doctrinal, la foi est pour le sujet indissociable du sentiment d'une présence, manifestée soudainement par l'illumination de la découverte, au cours du tâtonnement de l'exégète. La quête à la fois réglée et aveugle du lecteur croyant des Écritures, qui palpe et scrute les textes, joue un rôle analogue à celui de l'accomplissement de rites pour actualiser et rendre efficace le contrat avec les puissances supérieures dont l'existence est tenue pour assurée, mais dont l'identité reste hors d'atteinte.

Un ensemble de croyances à propos desquelles la réflexion d'Origène garde des traces, au cœur même d'un discours philosophique et monothéiste, des modèles de la religion grecque, ou peut être comparée, plus généralement, à des systèmes régentés par le polythéisme, est celui qui concerne les anges et les démons. Certes, dans sa réplique à Celse reprochant aux chrétiens de ne pas s'acquitter du culte dû aux êtres qui président aux joies et aux maux de cette vie, Origène inculque la vénération du seul Dieu Créateur et attribue à ses serviteurs les anges créés l'administration des biens utiles, en leur opposant les démons rebelles et pervertis, ceux auxquels Celse veut qu'on sacrifie. "On doit, écrit-il notamment, demander [à Dieu par JésusChrist] tout le secours et toute la protection de ses anges saints et justes, pour qu'ils nous arrachent aux démons terrestres absorbés dans la génération...» (Contre Celse, VIII,60, 1. 19-22) Et il distingue la recherche de l'intérêt gouvernant les honneurs religieux rendus aux démons tels que les conçoit Celse, de la consécration pure, par la piété et toutes les vertus, au Dieu de l'univers (voir tout le développement de VIII,55-65). Il reste qu'il croit en l'efficacité de prières rituelles pour régler les relations avec les puissances bonnes et mauvaises, de prières dont le sens n'est pas accessible. Il s'agit en effet de l'agencement de «noms barbares " présents dans les Écritures qui est doté d'un pouvoir sur les puissances invisibles. Le Contre Celse et l'Exhortation au martyre accordent à cette théorie une grande importance (Contre Celse, I,24-25 ; IV,33-34 ; 48; V,45-46; Exhortation 
au martyre, 46). D'après l'une des Homélies sur Josué21, l'arrangement sonore des noms hébreux a un sens intelligible pour les puissances invisibles, bonnes ou mauvaises, qui sont présentes dans les âmes, et il a sur elles un effet, fortifiant pour les unes, pernicieux pour les autres ${ }^{22}$. Cette théorie est attestée en dehors du christianisme au temps d'Origène, et il en fait luimême " une doctrine profonde et ineffable (Exhortation au martyre, 46). Elle est donc éminemment digne d'être reçue par les progressants, voire par ceux qui s'acheminent vers la perfection. La foi, en l'occurrence, supprime le clivage entre la croyance des simples et la "connaissance » réservée aux parfaits. C'est dans le même contexte, au cours de l'argumentation en faveur de cette doctrine, que s'efface la différence entre la religion et la magie, qu'Origène est amené alors à prendre au sérieux. Le savoir ésotérique retrouve, en les réinterprétant, des manières de concevoir et de pratiquer les relations avec les puissances invisibles qui n'ont pas besoin d'énoncer des doctrines. La foi en leur présence est entière, mais elle garde le silence sur leur identité et sur leur monde et elle fait confiance, pour la répartition des rôles, à la maîtrise de la coutume. On peut se demander si une telle attitude n'est pas susceptible d'un commentaire analogue à celui qu'inspire à Jean Pouillon (voir 1993, 30-36) la conduite de représentants d'une culture fort éloignée du christianisme et du temps d'Origène, en l'occurrence celle des Dangaleat du Tchad envers les génies que sont les margaï: on se risquerait à y voir aussi un service, ou un culte, qui ne comporte pas le passage par la croyance, c'est-à-dire par une "assertion malgré elle dubitative, relativisée », mais qui fait corps avec une expérience et s'impose comme efficace dès lors qu'il est correctement accompli ${ }^{23}$.

\section{Conclusion}

Soumettant à l'examen rationnel le contenu de la foi et s'efforçant de lui conférer la stabilité d'une connaissance, voire d'une science, Origène réintroduit en fait, sous la hiérarchie des "charismes ", une part d'indécision

21. XX, 1-2, texte conservé en grec dans la Philocalie, 12.

22. Une présentation succincte du thème est fournie par Le Boulluec (2003, 330-332).

23. Origène insiste sur la nécessité de proférer comme il convient les invocations des noms divins et les énoncés incantatoires (voir Contre Celse I,25, 1. 1-5; 1. 28-43). Il reste cependant que dans la préface du traité Des Principes, il met au nombre des sujets que «la prédication ecclésiastique » a enseignés l'existence des puissances adverses, qui est donc objet de foi. 
qui offre à chacun le pouvoir de déployer l'investigation nécessaire au geste de l'assentiment. Il ritualise en même temps le questionnement, sur le fond d'une croyance indubitable en l'existence de puissances invisibles, pour entrer en contact avec elles quand elles sont bonnes (l'Esprit saint, par un « toucher » constamment appliqué aux textes de l'Écriture, et les anges, par l'écoute et la "profération » de formules échappant à la communication ordinaire), pour les esquiver quand elles sont mauvaises (les démons, par des paroles apotropaïques). Une autre structure que celle de la foi érigée en savoir, régie peut-être par des lois plus générales du comportement religieux, au-delà du clivage entre doctrine et pratique rituelle, semble sousjacente au système de l'apologie chrétienne d'Origène.

\section{Références}

Benveniste, E. (1969), Le vocabulaire des institutions indo-européennes, Paris, Minuit.

Benz, E. et E. Klostermann, dir. (1935), Origenes Matthäuserklärung, Leipzig, Hinrichs (Die griechischen christlichen Schriftsteller der ersten drei Jahrhunderte 40. Origenes Werke 10).

Crouzel, H. (1971), Bibliographie critique d'Origène, Turnhout, Brépols.

- (1982), Bibliographie critique d'Origène. Supplément I, Turnhout, Brépols.

- (1996), Bibliographie critique d'Origène. Supplément II, Turnhout, Brépols.

Dorion, L.-A. (2002), "Aristote et l'invention de la dialectique", dans M. Canto-Sperber et P. Pellegrin, dir., Le Style de la pensée. Recueil de textes en hommage à Jacques Brunschwig, Paris, Les Belles Lettres, p. $182-220$.

FAHR, W. (1969), Theous nomizein. Zum Problem der Anfänge des Atheismus bei den Griechen, Hildesheim / New York, Olms (Spudasmata 26).

Guillaumont, A. (2004), Un philosophe au désert. Évagre le Pontique, Paris, Vrin.

Наdot, P. (2004), Le voile d'Isis. Essai sur l'histoire de l'idée de Nature, Paris, Gallimard.

HäLlström, G. af (1984), Fides simpliciorum according to Origen of Alexandria, Helsinki, Societas Scientiarum Fennica.

Harl, M. (1975), "La bouche et le cœur de l'apôtre. Deux images bibliques du "sens divin" de l'homme chez Origène ", dans Forma Futuri. Studi in 
onore del card. M. Pellegrino, Turin, Bottega d'Erasmo, p. 17-42. L'article est aussi paru dans M. HARL, Le déchiffrement du sens. Études sur l'herméneutique chrétienne d'Origène à Grégoire de Nysse, Paris, Institut d'Études augustiniennes (Études augustiniennes. Série Antiquité 135), 1993, p. 151-176.

- (1983), Origène, Philocalie 1-20. Sur les Écritures, Paris, Cerf (Sources chrétiennes 302).

Horn, H.J. (1993), «Zur Konzeption der Evidenz in der Schrift des Origenes Contra Celsum ", dans G.W. Most, H. Petersma et A.M. Ritter, dir., Philanthropia kai Eusebeia. Festschrift für Albrecht Dible zum 70. Geburtstag, Göttingen, Vandenhoeck \& Ruprecht, p. 183-198.

Lampe, G.W.H., dir. (1961), A Patristic Greek Lexicon, Oxford / New York, Clarendon.

Le Boulluec, A. (2003), "Le thème des noms divins chez les Pères aux III et IV ${ }^{e}$ siècles ", Annuaire de l'École Pratique des Hautes Études. Section des sciences religieuses, 110, p. 329-333.

LössL, J. (2002), «Der Glaubensbegriff des Klemens von Alexandrien im Kontext der hellenistischen Philosophie ", Theologie und Philosophie, 77, p. 321- 337.

Perrone, L. (1998), "Proposta per un commento: un'esemplificazione su Contro Celso I, 9-13 ", dans L. Perrone, dir., Discorso di veritá. Paganesimo, giudaismo e cristianesimo a confronto nel Contro Celso di Origene, Rome, Institutum Patristicum Augustinianum (Studia Ephemeridis Augustinianum 61), p. 225-256.

Pouillon, J. (1993), Le cru et le su, Paris, Seuil.

RAHNER, K. (1932), «Le début d'une doctrine des cinq sens spirituels chez Origène ", Revue d'Ascétique et de Mystique, 13, p. 113-145

Rist, J.M. (1981), "The Importance of Stoic Logic in the Contra Celsum ", dans H.J. Blumenthal et R.A. Markus, dir., Neoplatonism and Early Christian Thought. Essays in Honour of A.H. Armstrong, Londres, Variorum Publications, p. 64-78. L'article est aussi paru dans J.M. RIST, Platonism and Its Christian Heritage, London, Variorum Reprint, 1985, étude V.

Stoumsa, G.G. (1998), "Celsus, Origen and the Nature of Religion ", dans L. Perrone, dir., Discorso di veritá. Paganesimo, giudaismo e cristianesimo a confronto nel Contro Celso di Origene, Rome, Institutum Patristicum Augustinianum (Studia Ephemeridis Augustinianum 61), p. 81-94.

VeYne, P. (1983), Les Grecs ont-ils cru à leurs mythes?, Paris, Seuil. 


\title{
Résumé
}

Laissant de côté le pacte entre contractants de puissance inégale que supposent les termes pistis et pisteuein et l'élément quasi institutionnel qui établit la foi (pistis) sur une règle ecclésiastique, l'enquête s'applique à la réponse d'Origène au pamphlet du philosophe Celse contre le christianisme. À Celse qui réduit la foi chrétienne à la croyance et qui la soumet donc au doute, Origène prétend opposer les garants qui assurent sa validité, selon les méthodes de la persuasion rhétorique. Il l'éloigne de la croyance et de la coutume en lui octroyant un fondement «naturel ». Il est conduit aussi à admettre plusieurs degrés dans la connaissance religieuse, en modifiant la distinction opérée par Platon entre « foi » et « intellection ». L'exigence de compréhension et de savoir cependant n'exclut pas, dans la recherche du sens des Écritures comme dans les rapports avec les puissances invisibles, le recours à des pratiques de type rituel qui impliquent une expérience étrangère au régime de la croyance et de la foi.

\begin{abstract}
Putting aside the agreement between unequal contracting parties the words pistis and pisteuein imply and the quasi-institutional element which builds faith upon an ecclesiastical rule, this paper deals with the answer Origen gives to Celsus' pamphlet against Christianity. Whereas Celsus reduces Christian faith to belief and casts doubts upon it, Origen seeks to warrant its validity by the rhetorical art of persuasion. He moves it away from belief and custom by granting it a "natural» basis. He also admits that religious knowledge has several degrees and consequently modifies Plato's distinction between "faith» and "intellection». Prone as he is to require of faith science and certainty, he nevertheless resorts to some rituals in order to scrutinize the Scriptures or to deal with invisible powers in such a manner that experience prevails over belief and faith.
\end{abstract}

